\title{
A cathodoluminescence study on the diffusion length in AlGaInP/InGaP/AIInP solar cell heterostructures
}

\author{
Shabnam Dadgostar 1. Cantia Belloso Casuso ${ }^{1}$. Oscar Martínez ${ }^{1}$ Manuel \\ Hinojosa $^{2}$. Iván García ${ }^{2}$ Juan Jiménez¹.
}

Received: date / Accepted: date

\begin{abstract}
The diffusion length of minority carriers in a $p$-doped InGaP layer is derived from the cathodoluminescence (CL) intensity profiles. Two procedures are used. First, the CL profile is recorded along a line crossing the intersection between a thin metallic mask and the semiconductor, a second approach consists of the measurement of the intensity profile around an intentional scratch on the surface of the sample. A longer diffusion length is measured when using the metallic mask as compared to the scratch. We discuss the role of nonradiative recombination centers in the reduction of the diffusion length around the scratch. The temperature dependence of the diffusion length is also measured, it is found to decrease with temperature.
\end{abstract}

Keywords Minority Carriers · Diffusion Length · Optoelectronic Devices · Cathodoluminescence $\cdot$ III-V Multi-Junctions · III-V Solar Cell

\section{$1 \quad$ Introduction}

The ternary alloy $\operatorname{In}_{x} \mathrm{Ga}_{1-\mathrm{x}} \mathrm{P}$ is an interesting material that can be grown lattice-matched to GaAs for a composition of $\mathrm{In}_{0.49} \mathrm{Ga}_{0.51} \mathrm{P}$. It is used in different devices, e.g. light-emitting diodes, laser diodes,

\footnotetext{
${ }^{1}$ S. Dadgostar C. Belloso Casuso O. Martínez J. Jiménez

GdS-Optronlab Group, Dept. Física de la Materia Condensada, Universidad de Valladolid, Paseo de Belén, 19, 47011 Valladolid Spain

Tel.: +34-983-184956

Fax: +34-983-184956

E-mail: shabnam.dadgostar@uva.es

${ }^{2}$ M. Hinojosa I. García

Instituto de Energía Solar, ETSI de Telecomunicacíon, Universidad Politécnica de Madrid, 28040 Madrid, Spain
} 
photodetectors, and multi-junction solar cells. The efficiency of multi-junction solar cells based on this material exceeds $40 \%$ and is predicted to reach $50 \%$ in the near future [1-3].

Minority carrier transport properties are crucial for the operation of optoelectronic devices, therefore, the minority carrier diffusion length $\left(L_{D i f f}\right)$ is a parameter necessary to design and optimize these devices. The diffusion length depends on the band structure of the material, the crystal quality, alloy composition, doping concentration, and the presence of defects, either impurities or intrinsic. Cross-sectional electron beam induced current (EBIC) and transport imaging in a scanning electron microscope are two techniques applied to measure the minority carrier diffusion length. However, the electric fields and surface recombination are the challenges of the EBIC method [4,5], while a 2D-CCD camera is necessary for the transport imaging technique [6,7]. Cathodoluminescence (CL) has been also applied to measure the minority carrier diffusion length in semiconductors [8]. Two experimental approaches are used to determine the diffusion length [9]. In the first approach, a metallic mask is deposited on the semiconductor surface. This mask must be thin enough to permit the penetration of the electron beam across the metal reaching the semiconductor, where the electron-hole pairs are generated and recombined. The metal layer, however, must be thick enough to block the CL emission emerging out. The diffusion length is measured by scanning the e-beam along a line perpendicular to the metal/semiconductor border. When the e-beam is separated from the metal/semiconductor border a distance equal or shorter than the diffusion length, some carriers can reach the border recombining in the unmasked semiconductor region, and the emitted CL can be collected [10]. The second approach uses a defect, e.g. an isolated dislocation or other extended defects, to evaluate the diffusion length. Extended defects usually behave as nonradiative recombination centers. Around the defects, the CL intensity declines drastically due to the diffusion of carriers toward the defect, where they recombine non radiatively, giving a dark CL contrast. Therefore, the diffusion length might be estimated from the CL intensity profile collected around the defect $[11,12]$.

In this work, we estimate the lateral minority carrier diffusion length in a $p$-type $\operatorname{In}_{0.49} \mathrm{Ga}_{0.51} \mathrm{P}$ layer using the two approaches, a gold mask, and a scratch line respectively. The impact of the presence of defects is analyzed. 


\section{Experimental}

The samples used for measurements were lattice matched double AlGaInP/InGaP heterostructures deposited on a GaAs substrate $2^{\circ}$ miscut toward the (111) B plane. The growth was carried out in a horizontal low-pressure metalorganic vapor phase epitaxy (MOVPE) reactor (AIX 200/4). The precursors for the group-III elements are TMGa and TMIn, $\mathrm{AsH}_{3}$ and $\mathrm{PH}_{3}$ are used for the group-V, and $\mathrm{DTBSi}_{2}$, DETe and DMZn are the dopant precursors. The layer of interest is a $1000 \mathrm{~nm}$ thick $\operatorname{In}_{0.49} \mathrm{Ga}_{0.51} \mathrm{P}: \mathrm{Zn}$ sandwiched in between a $250 \mathrm{~nm}$ thick $\mathrm{Al}_{0.104} \mathrm{Ga}_{0.416} \mathrm{In}_{0.48} \mathrm{P}: \mathrm{Zn}$ layer and a $190 \mathrm{~nm}$ thick $\operatorname{In}_{0.49} \mathrm{Ga}_{0.51} \mathrm{P}: \mathrm{Si}$ layer. The nominal doping concentrations for $p$-type and $n$-type layers are $1 \times 10^{17} \mathrm{~cm}^{-3}$ and $1 \times 10^{18} \mathrm{~cm}^{-3}$ respectively. A scheme of the structure is shown in Fig.1(a).

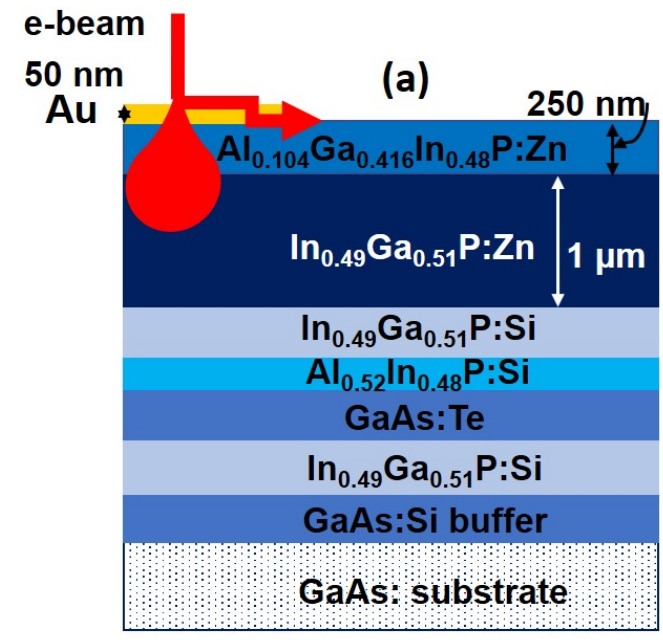

(c)

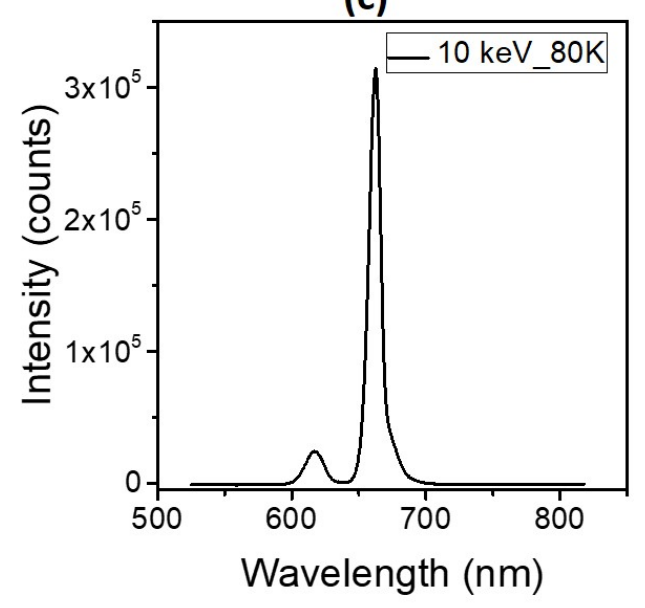

(b)

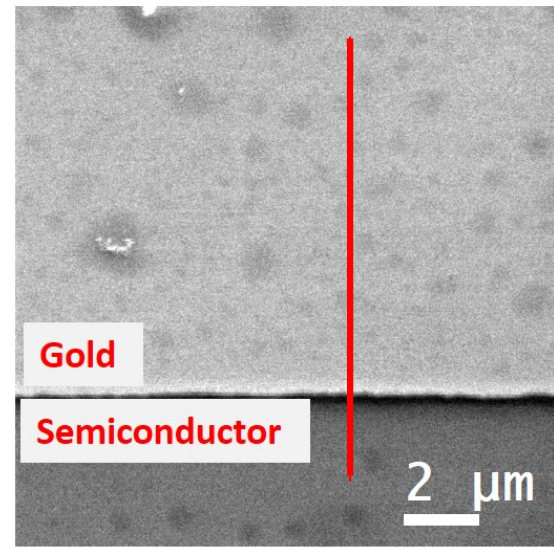

(d)

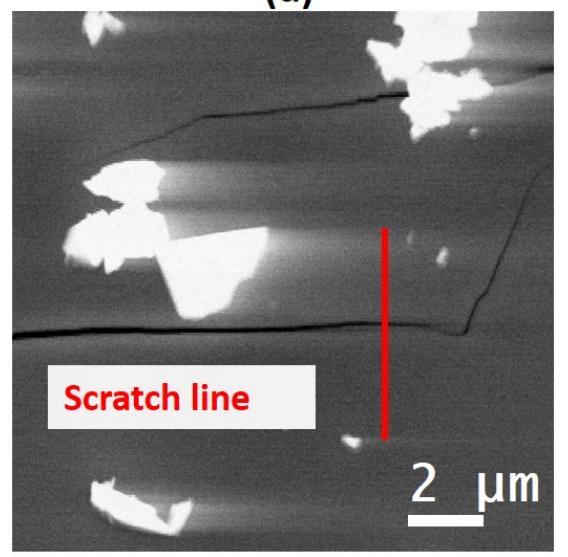

Figure 1(a) Scheme of the multi-junction (cross section). (b) To measure the diffusion length, the electron beam scanned a line normal to the gold/semiconductor border. The red line indicates the scan direction. (c) Typical recorded CL spectrum at $80 \mathrm{~K}$ for a $10 \mathrm{keV}$-beam energy. (d) SEM image of a scratch line with the scanning line.

The CL measurements were carried out in a LEO 1530 (Carl-Zeiss) field- emission scanning electron microscope (FESEM) equipped with a MonoCL2 (Gatan UK) CL system. The detection was done with a 
Peltier-cooled CCD detector. Fig.1(b) is a SEM image of the surface of the sample where one can see the gold/semiconductor border, the measurement was performed by scanning the beam transversally to the borderline (along the red line in Fig.1(b)) and recording the CL spectrum at each position, achieving an hyper-spectral line scan. The gold layer was $50 \mathrm{~nm}$ thick, which permits to excite the CL for beam energies above $10 \mathrm{keV}$ (Fig.2(b,c)). A typical CL spectrum is shown in Fig.1(c), the sharp peak at $660 \mathrm{~nm}(1.877$ $\mathrm{eV}$ at $80 \mathrm{~K}$ ) corresponds to the conduction $(\mathrm{CB})$ to valence band $(\mathrm{VB})$ emission in the InGaP layer, while the low intensity peak at $620 \mathrm{~nm}(2 \mathrm{eV})$ arises from the $\mathrm{CB}$ to $\mathrm{VB}$ transition in the AlGaInP top layer. The transmittance of the $50 \mathrm{~nm}$ thick gold layer in this spectral range is below $3 \%$ [13]. The thickness of the gold layer is a critical point of these measurements. One must look for a compromise between the thickness of the gold layer, and the e-beam energy (generation volume, and penetration depth). This problem is not present when one scans the e-beam across the scratch or any other extended defect, although in this case, one should take account of the size of the generation volume with respect to the defect. Fig.1(d) is the SEM image of the sample with a line scratch on its surface. The electron beam was scanned across the scratch line (along the red line) and the CL spectrum was recorded at each position. The e-beam energy was optimized in order to achieve the best conditions for the measurement of the diffusion length in both cases. The temperature was varied from 80 to $300 \mathrm{~K}$.

\section{$3 \quad$ Results and Discussions}

The diffusion length $\left(L_{D i f f}\right)$ was estimated for different e-beam energies $\left(E_{b}\right)$. The result is shown in Fig.2(d), where one observes the dependence of $L_{D i f f}$ with $E_{b}$, the measured diffusion length increases with $E_{b}$. The measured diffusion length at room temperature increases from $0.3 \mu \mathrm{m}$ under $7 \mathrm{keV}$ excitation to $1.4 \mu \mathrm{m}$ for $20 \mathrm{keV}$ excitation. This dependence is related to the generation volume and certainly to the carrier generation rate, in fact, the CL intensity measured shall be related to the flux of carriers reaching the metal border, which will be higher for higher carrier generation rate. One can establish a relation between $L_{\text {Diff }}$ and the maximum CL intensity $\left(I_{C L}\right)$, which depends on $E_{b}$. In fact, one observes that the measured $L_{D i f f}$ increases with $I_{C L}$, Fig.2(d). On the other hand, one must take account of the lateral dimension of the generation volume. The generation volumes for different $E_{b} S$ were calculated using open-access Casino software [14]. 
(a)

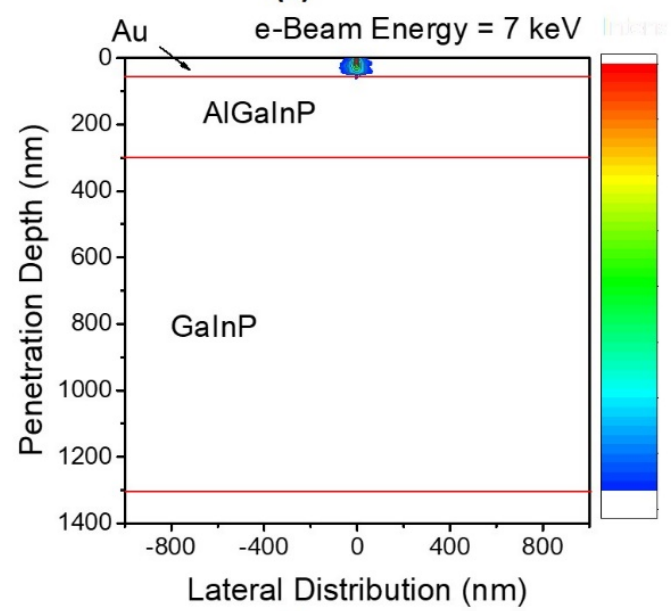

(c)

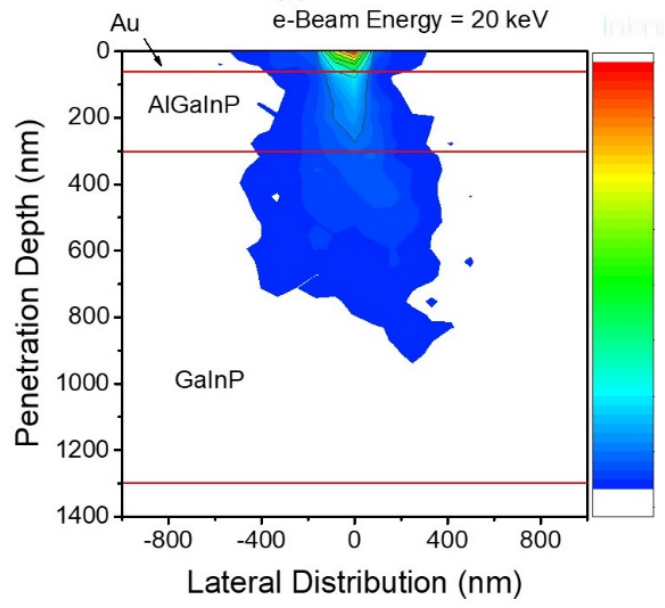

(b)

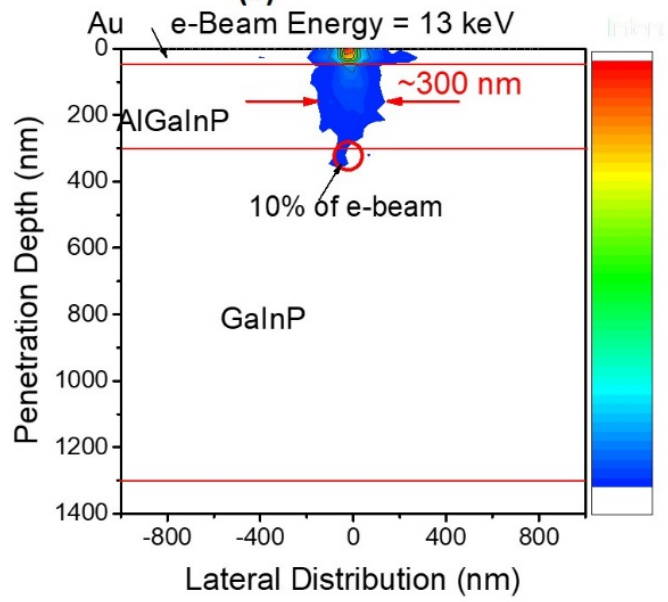

(d)

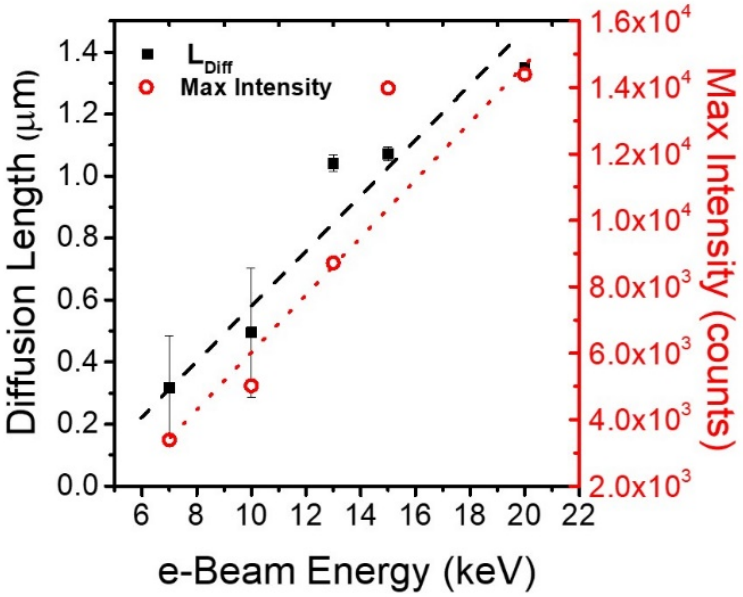

Figure 2 The Monte Carlo modeling of the absorbed energy cross-section for (a) $7 \mathrm{keV}$ and (b) $13 \mathrm{keV}$ and (c) $20 \mathrm{keV}$ e-beam energy ( the scale bar ranges from 0 to $100 \%$ energy loss). The bar indicates the absorbed energy from $5 \%$ (red) to 90\% (dark blue) (d) The evaluated diffusion length (black squares) at the gold/semiconductor intersection and the maximum recorded CL (red circles) for different e-beam energies. The black dash-line and red dot-line are eye guides respectively for $L_{D i f f}$ and Max Intensity.

For $E_{b}$ up to $15 \mathrm{keV}$, the generation volume is mainly located in the AlGaInP top layer, the carriers generated in this layer can diffuse laterally and recombine in the AlGaInP or the InGaP layers. They can also be drifted by the built-in potential from the top layer into the InGaP layer where they diffuse laterally and recombine giving the $660 \mathrm{~nm}$ peak. One should consider the two ways to reach the mask border in the InGaP layer [8]. In the first path, by lateral diffusion across the AlGaInP top layer, the surface recombination plays a major role, as revealed by very weak luminescence intensity emitted by the AlInGaP top layer. The role of the surface recombination is confirmed by the very short diffusion length measured from the $I_{C L}$ of the AlGaInP emission $(620 \mathrm{~nm})$. For low $E_{b}$, the carriers are generated in the top AlGaInP layer, Fig.2(a). The population of carriers reaching the InGaP layer is low and, therefore, the lateral flux 
of minority carriers that reaches the metal border is too low to give a significant CL intensity, therefore, the diffusion length is underestimated. As more electrons arrive into the InGaP layer, by increasing $E_{b}$, the lateral electron flux should increase enhancing the CL signal generated by the diffused electrons emerging out of the metal mask.

(a)

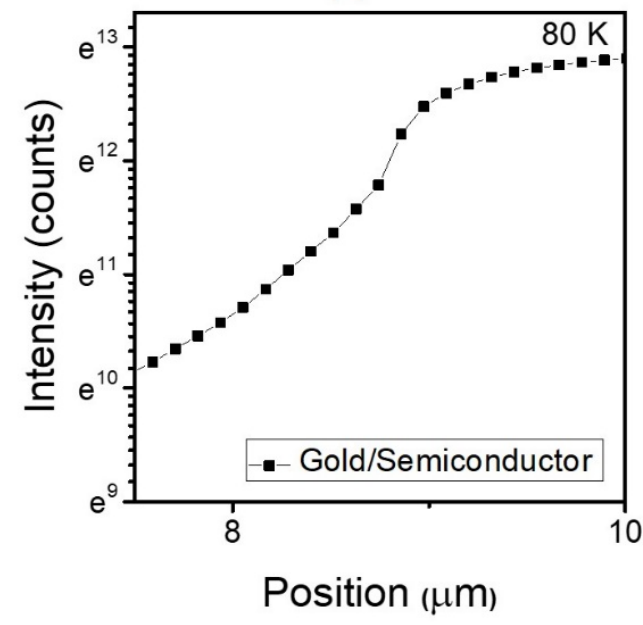

(b)

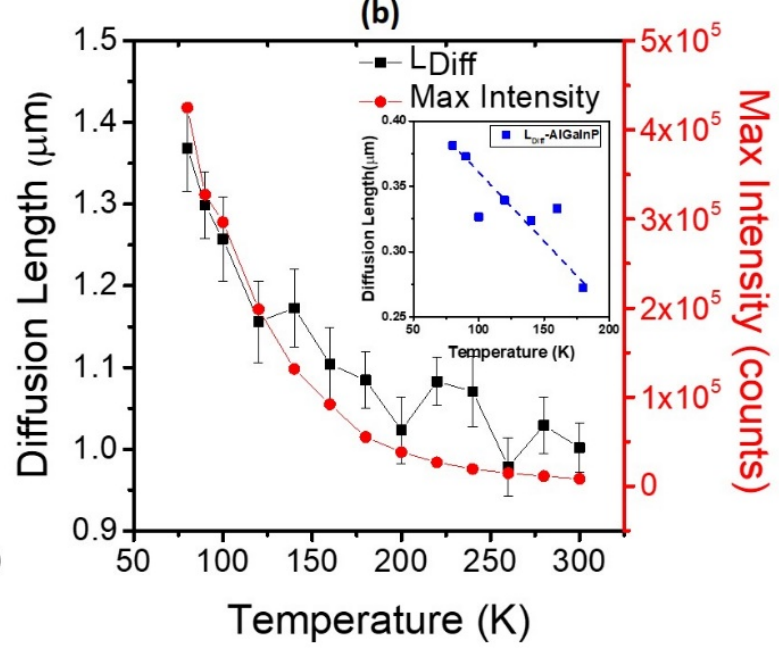

Figure 3 (a) Variation of CL intensity profiles at the border of gold/semiconductor at $80 \mathrm{~K}$ and under $13 \mathrm{keV}$ excitation energy and (b) measured diffusion length (black squares) and maximum CL intensity (red circles) at the gold/semiconductor intersection in the temperature interval of 80-300 K. The inset presents the temperature dependent diffusion length of minority carriers in the AlGaInP top layer. Lines are eye guides.

The primary electron range is the parameter limiting the spatial resolution; therefore, it must be considered when fitting the experimental data for obtaining the diffusion length from the CL intensity profile along a line scan crossing the metal/semiconductor border. One must take account of the electron range in the equation describing the CL intensity as a function of the distance of the e-beam to the metal mask edge (Fig.3(a)):

$$
I_{C L}=I_{0} \exp \left(\frac{-\left(x+r_{v}\right)}{L_{D i f f}}\right)
$$

where $L_{\text {Diff }}$ represents the diffusion length, $r_{v}$ is the excitation volume radius, and $x$ is the distance of the ebeam to the mask edge. To consider the impact of the generation volume, the lateral radius $r_{v}=0.15 \mu \mathrm{m}$ (taken at $90 \%$ energy loss) for $13 \mathrm{keV}$ was considered. The spectrum shape is preserved all along the scanning line, which permits to assume that there are not inhomogeneities associated with the presence of defects.

Fig.3(b) illustrates the variation of the diffusion length and CL intensity as a function of temperature. The results indicate the reduction of the diffusion length by increasing the temperature from $1.37 \mu \mathrm{m}$ at $80 \mathrm{~K}$ to 
about $1.0 \mu \mathrm{m}$ at room temperature. The surface recombination affects the diffusion length in the top AlGaInP layer. One can observe the $L_{D i f f}$ profiles of both the $660 \mathrm{~nm}(1.877 \mathrm{eV})$ band, corresponding to the InGaP layer, Fig.3(b), and the $620 \mathrm{~nm}(2 \mathrm{eV})$ band, corresponding to the AlGaInP top layer, Fig.3(b) (inset). The diffusion in the top layer is almost suppressed by the surface recombination, while the surface recombination will not contribute to the diffusion in the InGaP layer. Considering the relation between $L_{D i f f}$ and $T$ :

$$
L_{D i f f}=\sqrt{\frac{k_{B} T}{e} \mu \tau}
$$

where $\mu$ is the mobility, $\tau$ is the carrier lifetime, $k_{B}$ is the Boltzmann constant, and $T$ is the temperature, the decrease of $L_{D i f f}$ with $T$ should be due to a reduction of the $\mu \tau$ factor that overbalances the $T$ increase. One can expect a reduction of the lifetime, assuming the contribution of non-radiative recombination centers, on the other hand, a reduction of the mobility due to acoustic phonon scattering for increasing temperature in an alloy of these characteristics could account for such a decrease of the diffusion length [12]. The values obtained are on line with the values reported in recent works [15].

Alternatively, we studied the diffusion length by recording the CL spectra around a scratch. The scratch gives a dark contrast in the CL image because of the non-radiative recombination. The carrier lifetime in the neighborhood of the scratch is limited by the diffusion rate of the electrons towards the defect. The scratch line was not fully quenched, in fact the emissions from the top layer and the InGaP layer were still observed when the e-beam is on the scratch line, which accounts for a relatively low non radiative recombination activity, suggesting a low concentration of deep levels in the scratch. The e-beam was scanned transversally to the scratch line and the diffusion length was calculated from the exponential decay of the CL intensity as a function of the distance to the scratch line (Fig.4). The diffusion length estimated is shorter than the one measured when using the metallic mask. This reduction can be associated with the presence of nonradiative recombination centers surrounding the scratch, which had been created by the mechanical deformation induced by the scratch, also the band bending around the defect could contribute to drift the minority carriers toward the scratch where they shall recombine non radiatively. The nonradiative recombination centers would be responsible for a reduction of the effective carrier lifetime, which in the absence of surface recombination can be expressed as:

$$
\frac{1}{\tau_{e f f}}=\frac{1}{\tau_{n r}}+\frac{1}{\tau_{r}}
$$


Note that the two main recombination mechanisms deduced from the CL spectrum, are the CB-VB radiative recombination $(660 \mathrm{~nm})$, and non-radiative recombination at deep levels, as no other recombination channels are seen in the CL spectrum. On the other hand, we have seen that the surface recombination does not affect the emission of the InGaP layer. The role of the deep levels generated by the plastic deformation induced by the scratch is also supported by the asymmetrical CL profile at both sides of the scratch, which should be a consequence of the different distribution of the deep levels at both sides of the scratch line, Fig.4(a). Note that the band bending around the defect depends on the presence of deep levels capturing majority carriers, and under the electric field generated the minority carriers are captured, giving a shorter effective diffusion length. In the example of Fig. 4 this effect is relatively small; however, for deeper scratches generating more damage, one observes changes in the CL intensity profile around the scratch that are probably associated with the drift of the minority carriers by the electric field associated with the scratch.

(a)

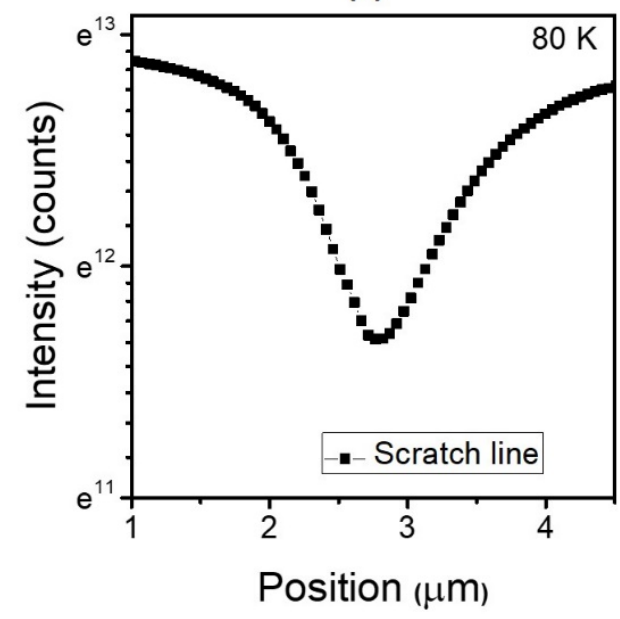

(b)

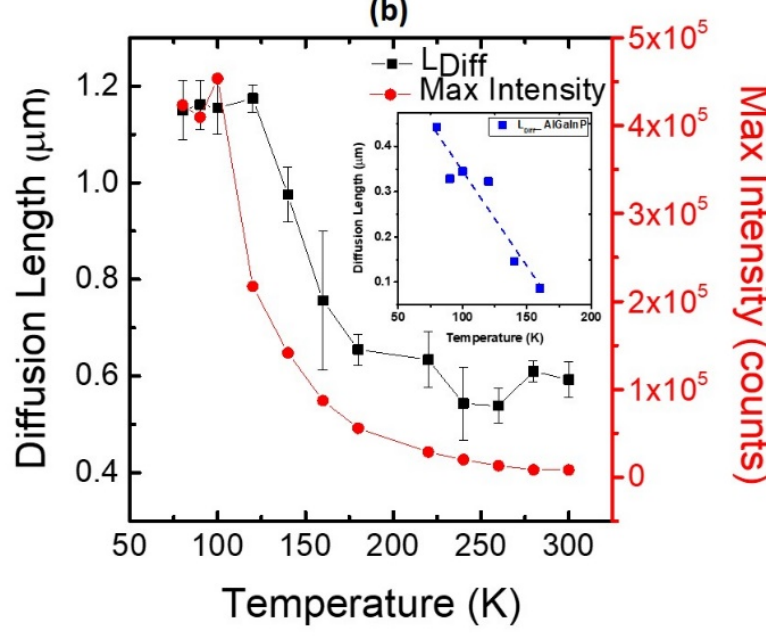

Figure 4 (a) CL intensity at the vicinity of scratch line under $13 \mathrm{keV}$ e-beam energy and $80 \mathrm{~K}$. (b) Temperature dependent diffusion length (black squares) and maximum CL intensity (red circles) at around the scratch line. The inset shows the temperature dependent diffusion length of minority carriers in the AlGaInP top layer. The lines are guides to the eye.

The temperature dependent results show the reduction of both CL intensity and diffusion length with increasing temperature. Here, the diffusion length decreases from $1.1 \mu \mathrm{m}$ to $0.6 \mu \mathrm{m}$ respectively at $80 \mathrm{~K}$ and $300 \mathrm{~K}$ (Fig.4(b)). This behavior suggests that the effective carrier lifetime is reduced with $T$, which is consistent with the presence of deep levels, for which the recombination rate increases with $T$. Therefore, one must be cautious with the estimation of the diffusion length, which can depend on the distribution of defects around the scratch or extended defect, e.g. a decorated dislocation, or a scratch surrounded by deep levels. 


\section{Conclusions}

We have presented a study of the in-plane minority carrier diffusion length in AlGaInP/InGaP heterostructures using spectrally resolved CL. The measurements were carried out on clean material using a metallic mask, and on damaged material using a scratch as the carrier sink. The experimental conditions, e-beam energy and temperature, were studied. The use of low energy e-beam leads to an underestimation of the diffusion length. This unattended result was discussed in terms of the low energy electron flux diffusing toward the mask edge under low excitation conditions.

The diffusion length around a scratch was shorter than the one measured across the metallic mask in clean material, which was explained in terms of the formation of deep levels that reduce the effective lifetime of the minority carriers and the electric field associated with the scratch.

Finally, the diffusion length was found to decrease with $T$ in the two cases suggesting that carrier lifetime is reduced by capture by deep levels and scattering by phonons is reducing the mobility.

Acknowledgements This work was supported by project VA283P18 (Junta de Castilla y Leon) and project ENE2017-89561-C4-3-R (Spanish Ministry of Economics and Competitiveness), and the European Fund for Regional Development (FEDER).

\section{References}

1. A. W. Bett, S. P. Philipps, S. S. Essig, S. Heckelmann, R. Kellenbenz, V. Klinger, M. Niemeyer, D. Lackner, and F. Dimroth, European photovoltaic solar energy conference; EU PVSEC 2013; 28th European Photovoltaic Solar Energy Conference and Exhibition; ed. by A. Mine, A. Jager-Waldau, P. Helm ; 1-6 ( 2013) ISBN:3936338337

2. F. Dimroth, T. N. D. Tibbits, M. Niemeyer, F. Predan, P. Beutel, C. Karcher, E. Oliva, G. Siefer, D. Lackner, P. Fus-Kailuweit, A. W. Bett, R. Krause, C. Drazek, E. Guiot, J. Wasselin, A. Tauzin, and T. Signamarcheix, IEEE J. Photovoltaics 6, 343 (2016).

3. M. Green, K. Emery, Y. Hishikawa, W. Warte, E. Dunlop, D. H. Levi, and A. W. Y. Ho-Baillie, IEEE Prog. Photovolt Res. Appl. 25, 3 (2017). 
4. M. Niemeyer, J. Ohlmann, A. W. Walker, P. Kleinschmidt, R. Lang, T. Hannappel, F. Dimroth, and D. Lackner, J. Appl. Phys. 122, 115702 (2017).

5. O. Marcelot, S. I. Maximenko, and P. Magnan, IEEE Trans. Electron Devices 61, 2437 (2014).

6. D. R. Luber, F. M. Bradley, N. M. Haegel, M. C. Talmadge, M. P. Coleman, and T. D. Boone, Appl. Phys. Lett. 88, 163509 (2006).

7. F. J. Schultes, T. Christian, R. Jones-Albertus, E. Pickett, K. Alberi, B. Fluegel, T. Liu, P. Misra, A. Sukiasyan, H. Yuen, and N. M. Haegel, Appl. Phys. Lett. 103, 242106 (2013).

8. D. Araújo, G. Oelgart, J. D. Ganière, and F. K. Reinhart, J. Appl. Phys. 76, 342 (1994).

9. J. Jimenez and J. W. Tomm, Spectroscopic Analysis of Optoelectronic Semiconductors (2016).

10. H. A. Zarem, P. C. Sercel, J. A. Lebens, L. E. Eng, A. Yariv, and K. J. Vahala, Appl. Phys. Lett. 55, 1647 (1989).

11. D. Araújo, G. Oelgart, J. D. Ganière, and F. K. Reinhart, Appl. Phys. Lett. 62, 2992 (1993).

12. N. Ino and N. Yamamoto, Appl. Phys. Lett. 93, 232103 (2008).

13. A. Axelevitch, B. Gorenstein, and G. Golan, Phys. Procedia 32, 1 (2012).

14. D. Drouin, a. R. Couture, D. Joly, X. Tastet, V. Aimez, and R. Gauvin, Scanning 29, 92 (2007).

15.F.J.Schultes, T. Christian, R.Jones-Albertus, E.Pickett, K. Alberi, B.Fluegel, T.Liu, P.Misra, A. Sukiasyan, H.Yuen, N.M.haegel; Appl. Phys. Lett. 103, 242106 (2013) 